DOI https://doi.org/10.30525/978-9934-26-019-3-3

\title{
AXIAL FORCE EFFECT ON THE OVERALL BUCKLING OF A COMPOUND REINFORCED SHELL STRUCTURE WITH THE POSITIVE GAUSSIAN CURVATURE AT AN EXTERNAL PRESSURE
}

\section{Gristchak V. Z., Dyachenko N. M.}

\section{INTRODUCTION}

The development of ways and means of creating reliable power shell structures in industrial, civil, chemical and aerospace engineering, and in other areas includes a significant range of theoretical, experimental, technological and computer-software problems. The creation of adequate mathematical models and methods of calculating the local and overall stability under combined loading, the study of new mechanical effects and phenomena that can significantly increase the bearing capacity of the developed structures and systems with a reduction in their material consumption should be related to these problems [1-17]. Improving the shell structure stability is achieved, in particular, by using the reinforcing elements in the form of longitudinal and transverse structural frames.

Under the prevailing action of an external pressure on a thin shell of rotation, the transverse reinforcement with intermediate rings is the most effective, and the discreteness consideration of their location allows us to offer rational stiffness characteristics of the structures being researched on the basis of studying the local and overall forms of buckling [1-5], and changes in the geometric shape of the middle surface [6-13].

As for compound shell systems, the results of studying the stability of a reinforced "cylinder-cone type" structure under the action of an external loading of various types, causing the loss of stability, should be noted here $[2,3,14-16]$. Some stability problems of the compound "barrel-ogive type" structure with a positive Gaussian curvature of the middle surface should be mentioned in [10]. On account of the behavior special features of the reinforced shell structures of this configuration, the solution method and the analysis results of the axial force effect on stability at a uniform external pressure are discussed in this paper. 


\section{Statement of the problem}

We consider the overall instability problem of a compound «barrelogive type» shell structure with a positive Gaussian curvature of the middle surface reinforced with the intermediate rings and consisting of two sections, in the elastic region of material deformation with thickness parameters $h$, elastic modulus $E$ and Poisson's ratio $v v$. The solution is built taking into account the discreteness of the reinforcing ring location under the combined loading with the uniform pressure $q$, which is normal for the middle surface, and axial (tensile or compressive) forces $T$ (Fig. 1).

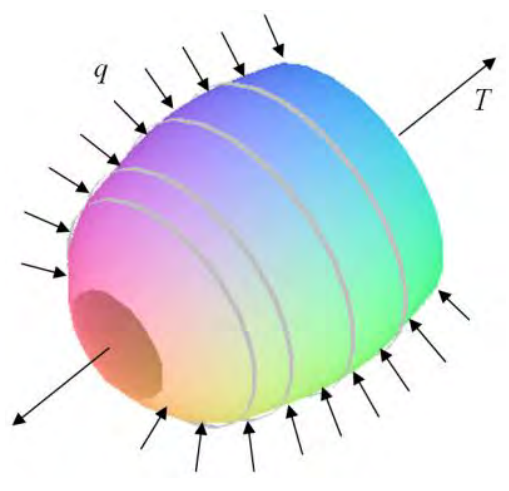

Fig. 1. Scheme of the shell structure

Under the action of axial compressive forces, buckling forms corresponding to the prevailing effect of the external pressure, which allows one with the axial tensile forces taken into account to accept the condition $[4,5]$ for the formation of one half-wave along the generatrix and $n$ waves in the annular direction in case of a structural instability, are considered. In such a case, for the class of "medium length" shells, the condition is $n^{2}>>1$

\section{The main relationships}

The coordinates are introduced along the generatrices of the cylindrical and conical surfaces $\bar{s}$ and $s$, respectively, the arc coordinate for the cylinder $-y$, the angular coordinate along the parallel of the cone $-\varphi$. 
A "barrel-shaped" shell is considered, and its surface of rotation has the following function of a parallel circle radius in a section which is perpendicular to the axis of rotation [10]:

$$
r=R\left(1+C_{b a r} \sin \frac{\pi \bar{s}}{L}\right),
$$

where $L$ and $R$ are the distance between the bases and the "barrel" base radius, $C_{b a r}$ is the relative height of the "barrel" generatrix camber.

The "ogive-shaped" shell of the middle surface, described by the function is considered

$$
r=\cos \alpha\left\lfloor s+C_{o g} l_{1} \sin \frac{\pi\left(s-l_{0}\right)}{l_{1}-l_{0}}\right\rfloor,
$$

where $l_{0}$ and $l_{1}$ are the distance along the axis $O s$ to smaller and larger bases, $\alpha$ is the taper angle, $C_{o g}$ is the relative height of the generatrix camber.

As following from [10], the resolving equations for each section of the compound structure with positive Gaussian curvature are obtained taking into account formulas (1), (2) and [12, 17]. In this case, the effects of tensile forces in the annular direction for the case of external compressive forces in a certain range, as well as the effects of compressive forces in the annular direction for the case of tensile forces in the meridional sections affecting the nature of the stability loss and the value of the critical external pressure forces are taken into account.

In keeping with [10], the differential equations of a basic stress-strain state as to the deflection functions for each compartment of the compound structure are:

$$
\begin{gathered}
a_{4}(\bar{x}) W_{b a r}^{I V}(\bar{x})+a_{3}(\bar{x}) W_{b a r}^{\prime \prime \prime}(\bar{x})+a_{2}(\bar{x}) \\
W_{b a r}^{\prime \prime}(\bar{x})+a_{1}(\bar{x}) W_{b a r}^{\prime}(\bar{x})+a_{0}(\bar{x}) W_{b a r}(\bar{x})=0, \\
b_{4}(x) W_{o g}^{I V}(x)+b_{3}(x) W_{o g}^{\prime \prime \prime}(x)+b_{2}(x) W_{o g}^{\prime \prime}(x)+b, \\
{ }_{1}(x) W_{o g}^{\prime}(x)+b_{0}(x) W_{o g}(x)=0
\end{gathered}
$$

where $\bar{x}=\bar{s} / L, x=s / l_{1}$, and the variable coefficients of the equations depend on the geometric characteristics of the shells and external loads. With the axial forces for the barrel-shaped section taken into account, the coefficients are determined by the formulas: 


$$
\begin{aligned}
& a_{0}=\pi^{3} n^{2} \varepsilon_{1} C_{b a r} K^{-2} \sin \pi \bar{x}\left(C_{b a r} \sin \pi \bar{x}+1\right)^{2} \eta_{b a r}- \\
& -\pi n^{2} \varepsilon_{1}\left(C_{b a r} \sin \pi \bar{x}+1\right)^{3} \chi_{b a r}+\pi n^{4} \varepsilon_{1}^{2} \omega^{-1}+ \\
& +2 \pi^{3} n^{2} \varepsilon_{1}^{2} C_{b a r} K^{-2} \omega^{-1}\left(C_{b a r} \sin ^{2} \pi \bar{x}-\sin \pi \bar{x}-2 C_{b a r}\right)+ \\
& +\pi^{5} C_{b a r} K^{-4}\left(C_{b a r} \sin \pi \bar{x}+1\right)^{3}\left[C_{b a r} \sin ^{2} \pi \bar{x}\left(C_{b a r} \sin \pi \bar{x}+1\right)+\right. \\
& \left.+n^{-2}\left(9 C_{b a r}^{2} \sin ^{3} \pi \bar{x}+8 C_{b a r} \sin ^{2} \pi \bar{x}-6 C_{b a r}^{2} \sin \pi \bar{x}+\sin \pi \bar{x}-4 C_{b a r}\right)\right] \text {; } \\
& a_{1}=K^{-2} \cos \pi \bar{x}\left(C_{b a r} \sin \pi \bar{x}+1\right)\left\{-K^{-2} \varepsilon_{1} C_{b a r}^{2} \pi^{4} \sin \pi \bar{x}\left(C_{b a r} \sin \pi \bar{x}+1\right)^{2} \eta_{b a r}+\right. \\
& +\varepsilon_{1} C_{b a r} \pi^{2}\left(C_{b a r} \sin \pi \bar{x}+1\right)^{3} \chi_{b a r}+2 \varepsilon_{1}^{2} n^{2} \omega^{-1} C_{b a r} \pi^{2}+ \\
& \left.+\pi^{4} K^{-2} C_{b a r}\left[\varepsilon_{1}^{2} \omega^{-1}\left(C_{b a r}^{2}-1\right)-2 n^{-2}\left(3 C_{b a r} \sin \pi \bar{x}+1\right)\left(C_{b a r} \sin \pi \bar{x}+1\right)^{3}\right]\right\} \text {; } \\
& a_{2}=K^{-2}\left(C_{b a r} \sin \pi \bar{x}+1\right)^{2}\left\{2 \varepsilon_{1} \pi\left(C_{b a r} \sin \pi \bar{x}+1\right) \eta_{b a r}+\right. \\
& +\varepsilon_{1} C_{b a r} \pi \sin \pi \bar{x}\left(C_{b a r} \sin \pi \bar{x}+1\right)^{2} \chi_{b a r}-2 \varepsilon_{1}^{2} n \omega^{-1} \pi+\pi^{3} K^{-2} C_{b a r} \times \\
& \times\left[\varepsilon_{1}^{2} \omega^{-1}\left(C_{b a r} \sin ^{2} \pi \bar{x}+2 \sin \pi \bar{x}+C_{b a r}\right)+2 n^{-2} \sin \pi \bar{x}\left(C_{b a r} \sin \pi \bar{x}+1\right)^{3}+\right. \\
& \left.\left.+3 n^{-4} \sin \pi \bar{x}\left(3 C_{b a r} \sin ^{2} \pi \bar{x}+\sin \pi \bar{x}-2 C_{b a r}\right)\left(C_{b a r} \sin \pi \bar{x}+1\right)^{2}\right]\right\} \text {; } \\
& a_{3}=2 \pi^{2} C_{b a r} n^{-4} K^{-4} \cos \pi \bar{x}\left(C_{b a r} \sin \pi \bar{x}+1\right)^{3} \times \\
& \times\left(3 C_{b a r}^{2} \sin ^{2} \pi \bar{x}+6 C_{b a r} \sin \pi \bar{x}+n^{4} \varepsilon_{1}^{2} \omega^{-1}+3\right) ; \\
& a_{4}=\pi n^{-4} K^{-4}\left(C_{b a r} \sin \pi \bar{x}+1\right)^{4}\left(C_{b a r}^{2} \sin ^{2} \pi \bar{x}+2 C_{b a r} \sin \pi \bar{x}+\varepsilon_{1}^{2} \omega^{-1} n^{4}+1\right),
\end{aligned}
$$

where

$$
\varepsilon_{1}=\frac{h}{R}, K=\frac{L}{R}, \omega=12\left(1-v^{2}\right), \chi_{b a r}=\frac{q R^{2}}{E h^{2}}, \eta_{b a r}=\frac{T}{2 \pi E h^{2}} ;
$$

the coefficients for the "ogive-shaped" section are determined as follows: 


$$
\begin{aligned}
& b_{0}=\left\lfloor\left(\pi^{2} C_{o g} \sin \Omega \cos ^{2} \alpha \eta_{o g}-\left(C_{o g} \sin \Omega+x\right)\left(1-K_{c}\right)^{2}\right) \chi_{o g}+\right. \\
& \left.+0,5 \pi^{2} \cos ^{2} \alpha C_{o g} \sin \Omega\left(K_{c}^{2}-x^{2}\right)\right] p^{3}\left(C_{o g} \sin \Omega+x\right)^{2}\left(1-K_{c}\right)^{2}+ \\
& +\delta^{2} \pi^{4} \cos ^{4} \alpha C_{o g}^{2} \sin ^{2} \Omega\left(C_{o g} \sin \Omega+x\right)^{4}+\delta \pi^{2} \cos ^{2} \alpha C_{o g}\left(C_{o g} \sin \Omega+x\right)^{3} \times \\
& \times\left[9 \pi^{2} C_{o g}^{2} \sin ^{3} \Omega+8 x \pi^{3} C_{o g} \sin ^{2} \Omega-4 \pi \cos \Omega\left(2 C_{c o n e} \sin \Omega+x\right)\left(1-K_{c}\right)+\right. \\
& \left.-\sin \Omega\left(\pi^{2}\left(6 C_{o g}^{2}-x^{2}\right)+2\left(1-K_{c}\right)^{2}\right)-4 x \pi^{2} C_{o g}\right]+p^{4}\left(1-K_{c}\right)^{4} \\
& b_{1}=\delta \pi^{2} \cos ^{2} \alpha C_{o g}\left(C_{o g} \sin \Omega+x\right)^{3}\left(1-K_{c}\right)\left[p \varepsilon \sin \Omega\left(K_{c}-1-\pi C_{o g} \cos \Omega\right) \eta_{o g}+\right. \\
& \left.+2\left(C_{o g} \sin \Omega+x\right)\left(1,5 \pi C_{o g} \sin 2 \Omega+2 \sin \Omega\left(1-K_{c}\right)+\pi x \cos \Omega\right)\right] \\
& b_{2}=\left(C_{o g} \sin \Omega+x\right)^{3}\left(1-K_{c}\right)^{2}\left\{\left(1-K_{c}\right)^{2}\left(p^{2} \eta_{o g}+6\left(C_{o g} \sin \Omega+x\right)\right)+\right. \\
& +3 C_{o g} \pi\left(C_{o g} \sin \Omega+x\right)\left(\pi\left(2 C_{o g}-3 C_{o g} \sin { }^{2} \Omega-x \sin \Omega\right)+4 \cos \Omega\left(1-K_{c}\right)\right)- \\
& \left.-2 \delta \pi^{2} \cos ^{2} \alpha C_{o g} \sin \Omega\left(C_{o g} \sin \Omega+x\right)^{2}\right\} \\
& b_{3}=6\left(C_{o g} \sin \Omega+x\right)^{5}\left(1-K_{c}\right)^{3}\left(\pi C_{o g} \cos \Omega+1-K_{c}\right) \\
& b_{4}=\left(C_{o g} \sin \Omega+x\right)^{6}\left(1-K_{c}\right)^{4} .
\end{aligned}
$$

where

$$
\begin{gathered}
\delta=\frac{n^{2}}{\cos ^{2} \alpha}, p=\varepsilon \delta, \varepsilon=\sqrt{\frac{h \operatorname{ctg} \alpha}{l_{1} \sqrt{12\left(1-v^{2}\right)}}}, K_{c}=\frac{l_{0}}{l_{1}}, \chi_{o g}=\frac{q l_{1}}{E h \varepsilon^{3} \operatorname{tg}^{3} \alpha}, \\
\eta_{o g}=\frac{T \cos \alpha}{2 \pi E h \varepsilon^{2} l_{1} \sin ^{3} \alpha} .
\end{gathered}
$$

The specifics of applying the finite difference method [18] for solving equations (3), (4) are described in [10], and the matrix algorithm for the discreteness consideration of the intermediate ring location is described in $[4,5]$ :

$$
\begin{aligned}
W_{l e f t}\left(t_{1}\right) & =W_{r i g h t}\left(t_{2}\right), & W_{l e f t}^{\prime}\left(t_{1}\right)=W_{r i g h t}^{\prime}\left(t_{2}\right), \\
W_{l e f t}^{\prime \prime}\left(t_{1}\right)+G_{c y l / c o n e, 2}^{*} W_{l e f t}^{\prime}\left(t_{1}\right) & =W_{r i g h t}^{\prime \prime}\left(t_{2}\right), & W_{l e f t}^{\prime \prime \prime}\left(t_{1}\right)-G_{c y l / c o n e, 1}^{*} W_{l e f t}\left(t_{1}\right)=W_{r i g h t}^{\prime \prime \prime}\left(t_{2}\right),
\end{aligned}
$$


where $t_{1}$ and $t_{2}$ are the coordinates of the section conjugation, $G_{c y l / c o n e, 1}^{*}$ and $G_{c y / / c o n e, 2}^{*}$ are the dimensionless stiffness parameters of the rings placed on the corresponding (locally cylindrical or locally conical) shell both in the plane of initial curvature and from its plane are defined by the formulas:

$$
\begin{gathered}
G_{c y l, 1}^{*}=G_{1} \frac{R^{3}}{r_{\text {ring }}^{3}}, G_{c y l, 2}^{*}=G_{2} \frac{R^{3}}{r_{\text {ring }}^{3}}, \\
G_{\text {cone }, 1}^{*}=G_{1} \frac{R^{3} L_{\text {cone }}}{\cos ^{2} \beta r_{\text {ring }}^{4}}, G_{\text {cone }, 2}^{*}=G_{1} \frac{R^{3} L_{\text {cone }}}{r_{\text {ring }}^{4}}, L_{\text {cone }}=\frac{R}{\cos \beta} \\
G_{1}=\frac{n^{4}\left(n^{2}-1\right)^{2}(E J)_{x}^{\text {ring }}}{E h R^{3}}, \quad G_{2}=\frac{n^{2}\left(n^{2}-1\right)^{2}(E J)_{z}^{\text {ring }}}{E h R^{3}\left(n^{2}+1\right)} .
\end{gathered}
$$

Here $J_{x}^{\text {ring }}, J_{z}^{\text {ring }}$ are the moments of inertia under bending the ring in the plane of the initial curvature and, accordingly, from its plane, $r_{\text {ring }}$ is the radius of the ring, $\beta$ is the base angle of the "local cone".

\section{Results}

A hinge-supported compound shell structure with the following characteristics is considered as an example: $h=0,3 \mathrm{~cm}$, $E=7 \cdot 10^{5} \mathrm{~kg} / \mathrm{cm}^{2}, \quad v=0,32$. The "ogive-shaped" compartment is selected with the following parameters: $l_{1}=182 \mathrm{~cm}, l_{0}=0,45 l_{1}, \quad \alpha=75^{\circ}$, and the "barrel-shaped" section is selected with the parameters as follows: $L=2,5 R$.

The algorithm of determining the equal stability of the compound structure sections relative to the geometric and stiffness characteristics and the nature of the external loading corresponds to $[2,3,10]$. We consider both hinge-supported sections ("barrel" and "ogive") taken individually, and the compound structure as a whole. The equal values of critical external pressure correspond to the sections correlated in terms of geometric and stiffness characteristics with relative camber parameters $C_{b a r}$ and $C_{o g}$. For specified geometric and stiffness characteristics and curvature parameters of the section meridian $C_{o g}=0,062596, C_{b a r}=0,137$, the critical external pressure values of the local and overall buckling forms are $q_{b a r}=q_{o g} \approx 2,97 \mathrm{~kg} / \mathrm{cm}^{2} \quad$ and $\quad q_{\text {constr }}=2,3 \mathrm{~kg} / \mathrm{cm}^{2}$ respectively. 
In such a case, the intermediate ring on the "ogive-shaped" shell is positioned so that the sections into which the conical shell is partitioned are equally stable. The location of the ring in the controlling case is determined by the ratio $L_{\text {left }}: L_{\text {right }}=1,809: 1$ (from smaller base of the conical compartment).

Dimensionless parameters for the external load characteristics are introduced

$$
\widehat{T}_{\text {bar } / o g}=\frac{T}{T_{\text {classic, cyl/cone }}}, \quad \widehat{q}_{\text {bar } / o g}=\frac{q}{q_{\text {classic, } \text { cyl/cone }}}, T^{*}=\frac{T}{E h_{0}^{2}}, q^{*}=\frac{q}{q_{\text {constr }}} .
$$

Here are determined the classical values of critical loads for cylindrical and conical shells by the dependencies given in [17].

Figures 2-6 show the results of numerical calculations of the critical external pressure forces for the shell structures under study, depending on the nature of loading by axial forces, the parameters of the meridian camber $C_{b a r / o g}$, the stiffness of the rings $G_{1,2}$, the amount of rings in accordance with the presented schemes and the nature of the wave formation in case of the loss in stability according to the minimization of critical forces in a wavenumber $n$.

The action of the compressive forces on shells with zero Gaussian curvature (of a cylindrical or conical form) leads to a decrease in the critical external pressure, and the action of the tensile ones leads to an increase. The result is a decreasing curve with the dependence of the critical pressure on the axial force, separating the stability region from the instability region (a blue line in Figs. 2 and 3). If the meridians of the middle surface of the "barrel" (Figs. 2 and 4) and "ogive" type shells (Figs. 3 and 5) have a positive Gaussian curvature, then a maximum point exists on the curves of the indicated dependencies. In this case, either the compressive or tensile axial force may correspond to the highest critical pressure value depending on the relative camber of the "barrel-shaped" or "ogive-shaped" compartment. 


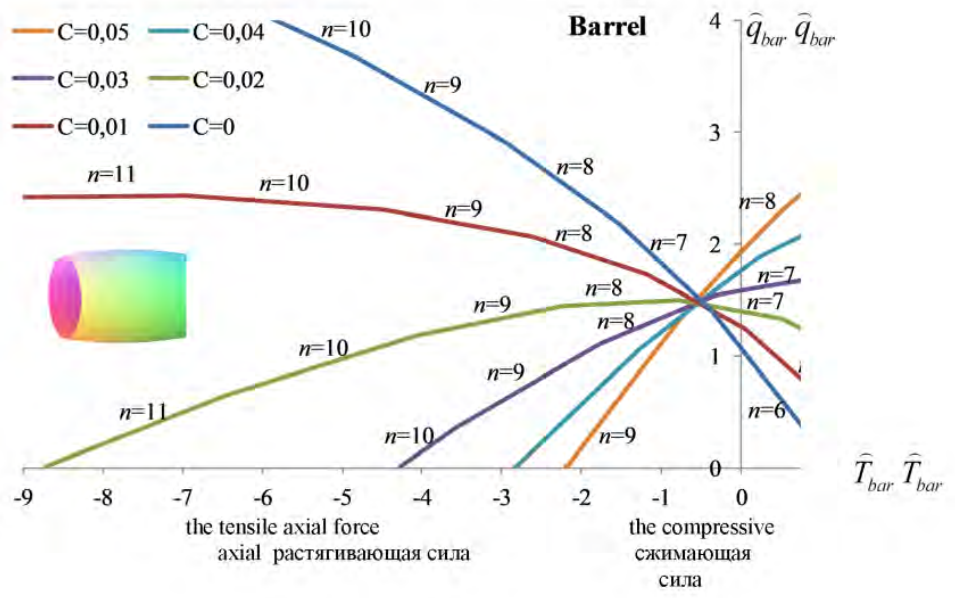

Fig. 2. The effect of axial forces on the critical external pressure value of the unreinforced "barrel-shaped" shell

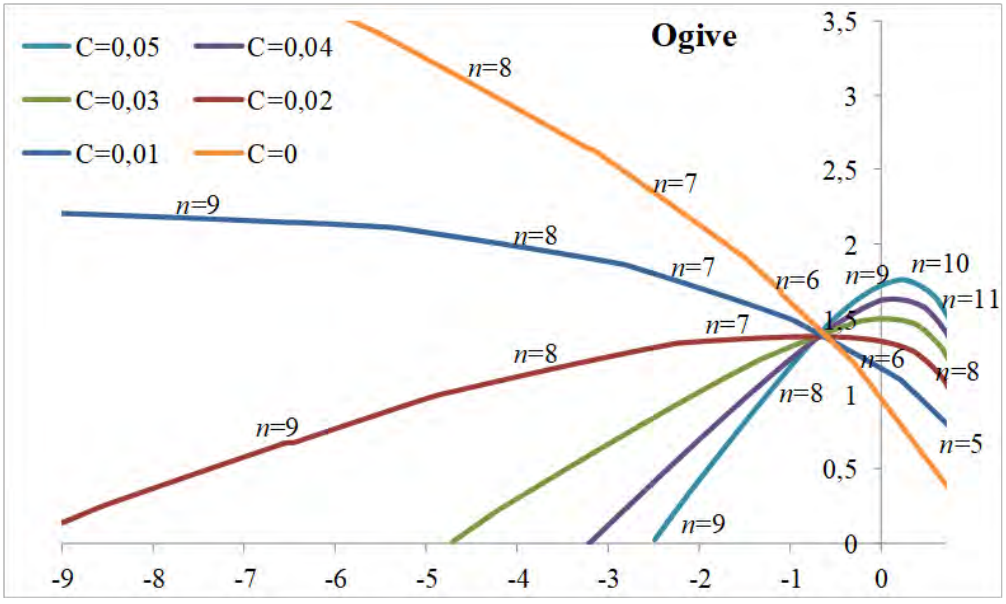

Fig. 3. The effect of axial forces on the stability of the reinforced "barrel-shaped" shell 


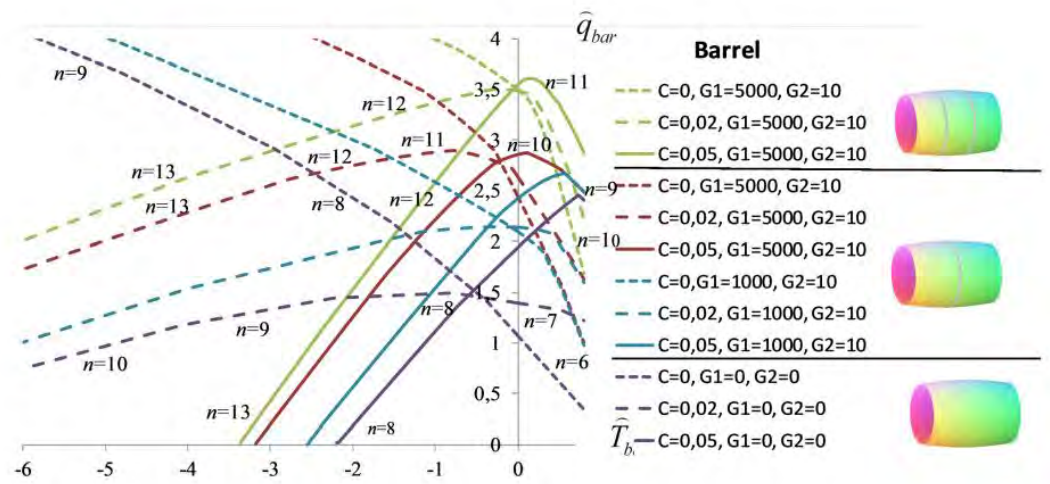

Fig. 4. The effect of axial forces on the stability of the reinforced "barrel-shaped" shell

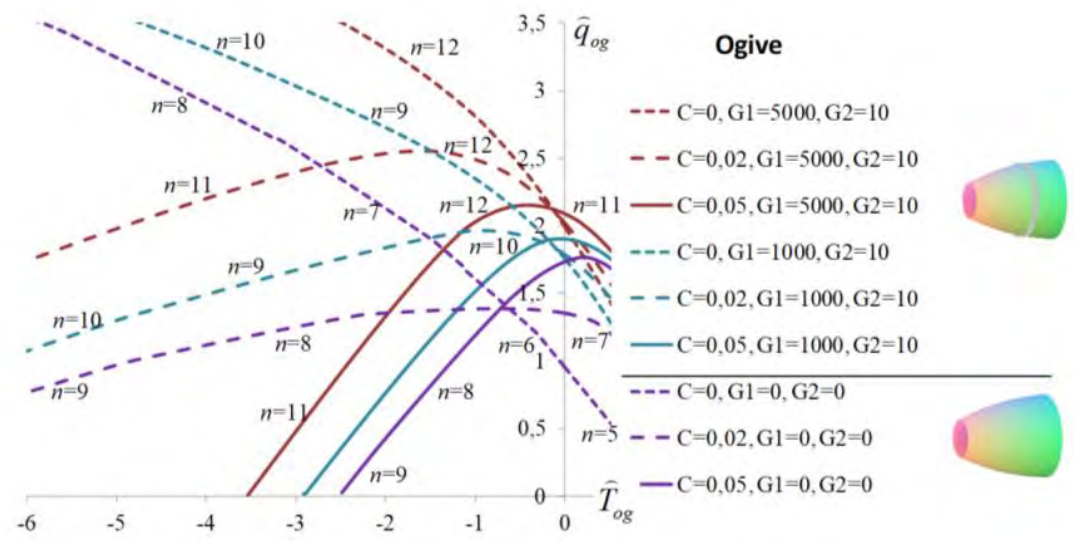

Fig. 5. The effect of axial forces on the stability of the reinforced "ogive-shaped" shell at the external pressure 


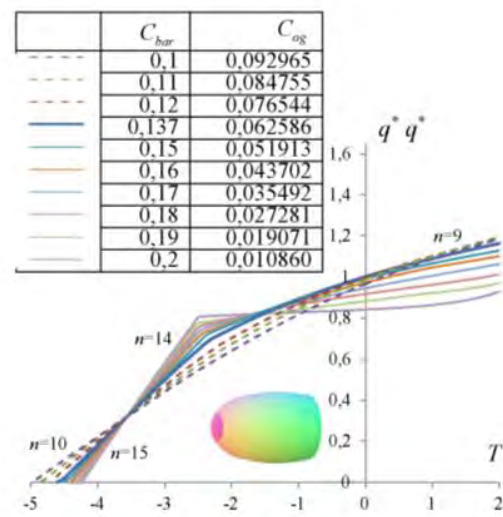

a

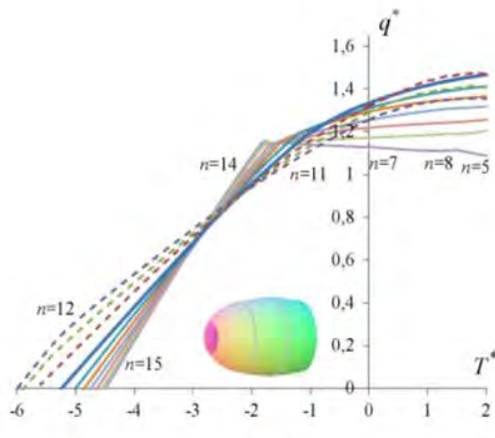

c

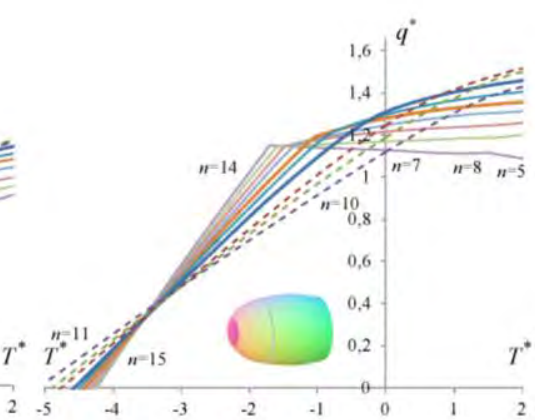

b

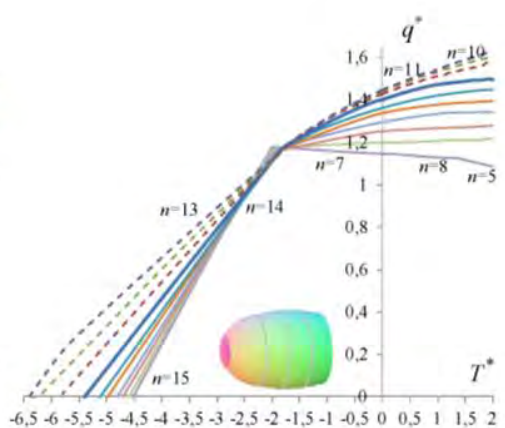

d

Fig. 6. The effect of the meridian curvature of the compartments on the stability of the compound shell structure of the "barrel-ogive" type at the external pressure: $a$ - unsupported; $b$-d - reinforced with one (b), two (c) or three (d) stiffness rings $G_{1}^{*}=5000, G_{2}^{*}=10$

For convex shells of all studied types, the tensile force leads to a decrease in the critical external pressure up to its zero value (Fig. 2-7). The inversion effect of the meridian camber of the middle surface under the action of the tensile force characterized by the intersection point of the constructed dependences is noted. For unreinforced structures of the "barrel" and "ogive" type, the corresponding inversion points are presented in Fig. 2 and 3, and for reinforced ones these points are shown 
in Fig. 4 and 5. The inversion points in Fig. 4 and 5 are illustrated by the intersection of lines of the same color, which correspond to the equal values of stiffness characteristics. For compound "barrel-ogive type" structures, the inversion effect is shown in Fig. 6. The location of the inversion points depends on the nature of the reinforcement and the value of the ring stiffness parameters. In Fig. 6, the bold lines show the dependencies corresponding to the equally stable structure. In Fig. 7, the presented dependencies characterize the stiffness effect of the equally stable structure on its behavior under the combined loading.

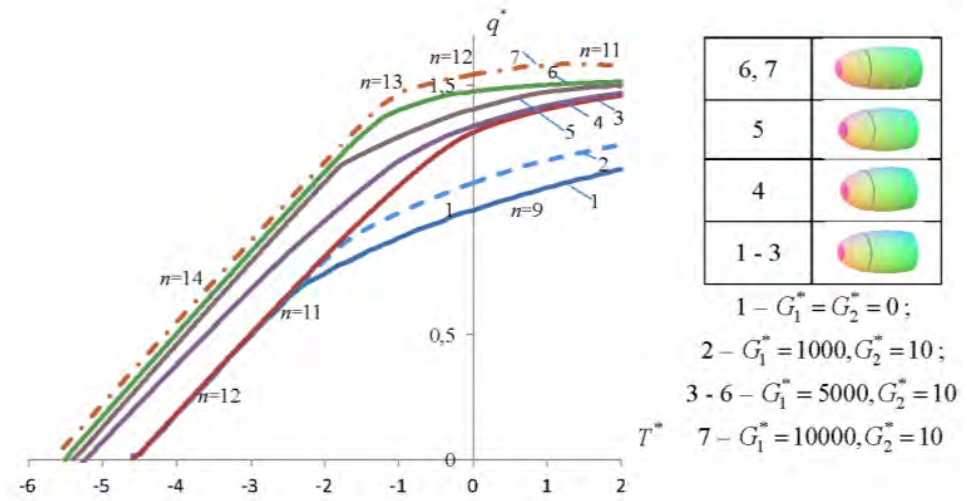

Fig. 7. The effect of axial forces on the stability of the reinforced «barrel-ogive» type shell structure with camber parameters $C_{o g}=\mathbf{0 , 0 6 2 5 8 6 ,} C_{b a r}=0,137$ at the external pressure

The inversion points in Fig. 4 and 5 are illustrated by the intersection of lines of the same color, which are correspond to equal values of stiffness characteristics. For compound "barrel-ogive" structures, the inversion effect is shown in Fig. 6. The location of the inversion point depends on the nature of the reinforcement and the magnitude of the rigidity parameters of the rings. In Fig. 6, the bold lines show the dependencies corresponding to the equally stable construction. In fig. 7, the presented dependences characterize the influence of rigidity of an equally stable structure on its behavior under combined loading. 


\section{CONCLUSIONS}

The results of the study for the compound shell structures with a positive Gaussian curvature reinforced with rings with the combined action of axial forces and external pressure have shown that while designing real structures, the change of the meridian curvature from the center of curvature and the presence of compressive longitudinal forces can lead to a significant increase in the critical external pressure by 1.5-2 times compared to the shells with zero Gaussian curvature, that is associated with the appearance of tensile annular stresses at the initial stage of loading due to the axial forces and the "snapping" effect on account of the middle surface curvature along the meridian.

When loading a compound shell structure with tensile forces, the effect of "inversion of the meridian curvature parameter of the middle surface for the shell of rotation" on the critical external pressure was found, this must be taken into account in practical calculations of shell structures operating on stability. The approach discussed in the paper makes it possible to estimate the critical loads of local and overall forms of buckling with an estimate of the stiffness characteristics of the reinforcing elements, which ensure equal stability of the spans of a compound shell structure.

The veracity of the obtained results is confirmed by the following factors. Firstly, they correspond to the physical representation of the deformation nature of systems under study. Secondly, in limiting cases, the numerical data obtained satisfactorily corresponds to the results based on other methods. In structures with zero Gaussian curvature, the critical pressure values obtained in the study deviate from the results of $[5,17]$ by less than $10 \%$.

\section{SUMMARY}

We consider the problem of overall instability of the compound rotational shell structure with a positive Gaussian curvature of the middle surface reinforced with transverse stiffeners (rings) associated with the effect of the meridian curvature and axial compressive or tensile forces at the uniform external pressure. Particular attention is paid to the shell structure of the «barrel-ogive» type with the analysis of local, relative to the reinforced compartments, and overall forms of structural instability as a whole including the intermediate rings under the combined loading.

The solution of the basic differential stability equations is based on a "semi-momentless" theory of thin medium length shells on the premise that the variability nature of the stress-strain state of the shell in one of 
the directions, in the considered case it is in the circumferential direction, significantly exceeds the variability of the state in the orthogonal direction.

In this problem, a sinusoidal approximation of the meridian form of the constituent shell elements is used, and the effect of the location discreteness and stiffness characteristics of the reinforcing rings is also taken into account. On the basis of the studies, a significant effect of the meridian curvature parameter of the middle surface and axial compressive forces on the stability of the structure under the combined loading is noted. The determined "inversion effect" of the axial tensile force action relative to the meridian curvature parameter on the stability and critical force value of the shell structures under study at the external pressure is discussed.

\section{REFERENCES}

1. X., Bai, W., Xu, H., Ren and J., Li, "Analysis of the influence of stiffness reduction on the load carrying capacity of ring-stiffened cylindrical shell," Ocean Engineering, vol. 135, pp. 52-62, May 2017, doi: 10.1016/j.oceaneng.2017.02.034.

2. P. G., Degtyarenko, V. Z., Gristchakand N. M., Dyachenko, “ To the stability calculation of a combined shell structure taking into account the discreteness location of the intermediate rings," Problems of computational, mechanics and strength of structures, vol. 29, pp. 113-131, 2019, doi: 10.15421/42190010.

3. P. G., Degtyarenko, V. Z., Gristchak, D. D., Gristchak and N. M., Dyachenko, "To equistability problem of the reinforced shell structure under combined loading," Space science and technology-Kosmicna nauka i tehnologia, vol. 25, no. 6 (121), pp. 3-14, 2019, doi: 10.15407/knit2019.06.003.

4. V. Z., Gristchak and A. I., Manevich, "Influence of a ring stiffness on a bend out of a plane on the stability of a reinforced cylindrical shell," Gidroaeromekhanika i teoriya uprugosti, issue 14, pp. 121-130, 1972

5. I. N., Preobrazhenskii and V. Z., Gristchak, Stability and oscillations of conical shells, Moskow: Mashinostroenie, 1986.

6. M., Amabili, "Nonlinear vibrations and stability of laminated shells using a modified first-order shear deformation theory," European journal of mechanics A-Solids, vol. 68, pp. 75-87, March-April 2018, doi: 10.1016/j.euromechsol.2017.11.005. 
7. M., Andres and R., Harte, "Buckling of concrete shells: A simplified numerical approach," Journal of the International Association for Shell and Spatial Structures: IASS, vol. 47, no. 3, n. 152, Desem. 2006. [Online]. Available: http://citeseerx.ist.psu.edu/viewdoc/ download?doi $=10.1 .1 .550 .2773 \&$ rep $=$ rep1\& type $=$ pdf.

8. K.V., Avramov, M.V., Chernobryvko, O., Kazachenko and T.J., Batutina, "Dynamic instability of parabolic shells in supersonic gas stream," Meccanica, vol. 51, pp. 939-950, July 2015, doi: 10.1007/s11012-015-0247-4.

9. X., Bai, R., Tang, Y., Zan and J., Li, "Stability analysis of a cylindrical shell with axially symmetric defects under axial compression based on the reduction stiffness method," Ocean Engineering, vol. 193, no. 106584, Desem. 2019, doi: 10.1016/j.oceaneng.2019.106584.

10.P. G., Degtyarenko, V. Z., Gristchak and N. M., Dyachenko, "Statement and basic solution equations of the stability problem for the shell-designed type "barrel-revived" under external pressure," Problems of computational, mechanics and strength of structures, vol. 30, pp. 33-52, 2019, doi: 10.15421/4219025.

11. M. A., Iqbal, G., Tiwari, and P. K., Gupta, "Energy dissipation in thin metallic shells under projectile impact," European Journal of Mechanics - A/Solids, vol. 59, Sept.- pp. 37-57, Oct. 2016, doi: 10.1016/j.euromechsol.2016.03.004

12.N. V., Kolkunov, Osnovy rascheta uprugih obolochek, Moskow: Vyssh. shkola, 1972.

13.H., Schmidt, "Two decades of research on the stability of steel shell structures. Available at the University of Essen (1985-2005): Experiments, evaluations, and impact on design standards," Advances in Structural Engineering. Special Issue for Prof. Ratter, pp. 1-29, March 2018, doi: 10.1177/1369433218756273.

14.D. V., Akimov, V. Z., Gryshchak, S. I., Gomenyuk, I. F., Larionov, D. V., Klimenko and V. N., Sirenko, "Finite-element analysis and experimental investigation on the strength of a three-layer honeycomb sandwich structure of the spacecraft adapter module," Strength of materials, vol. 48 no. 3, pp. 379-383, Aug. 2016, doi: 10.1007/s1 1223-016-9775-y.

15.J. G., Teng and M., Barbagallo, "Shell restraint to ring buckling. Available at cone-cylinder intersections," Engineering Structures, vol. 19, issue 6, pp. 425-431, 1997, doi: 10.1016/S0141-0296(96)00087-9.

16. Y, Zhao and J. G., Teng, "A stability design proposal for conecylinder intersections under internal pressure," International Journal of 
Pressure Vessels and Piping, vol. 80, issue 5, pp. 297-309, May 2003, doi: 10.1016/S0308-0161(03)00048-6.

17.A. S., Volmir, Ustojchivost' deformiruemykh sistem, Moskow: Nauka, 1967.

18.E., Kamke, Spravochnik po obyknovennym differenczial`nym uravneniyam, Moskow: Nauka, 1971.

\section{Information about the authors:}

Gristchak V. Z.,

Doctor of Technical Sciences, Professor, Head of the Department of Applied Mathematics and Mechanics,

Zaporizhzhia National University 66, Zhukovskoho str., Zaporizhzhia, 69600, Ukraine

Dyachenko N. M.,

Candidate of Physical and Mathematical Sciences $(\mathrm{PhD})$, Associate Professor at the Department of Fundamental Mathematics,

Zaporizhzhia National University 66, Zhukovskoho str., Zaporizhzhia, 69600, Ukraine 